Vladimir Rubilar Rivera - Fundamentos para realizar estudios...

\title{
FUNDAMENTOS PARA REALIZAR ESTUDIOS E INVESTIGACIONES JURÍDICAS CONJUNTAS ENTRE ESCUELAS DE DERECHO LATINOAMERICANAS. SUGERENCIAS EN EL MARCO DE LA INTERNACIONALIZACIÓN DE LAS UNIVERSIDADES
}

\author{
Vladimir Rubilar Rivera
}

Abogado

Profesor de Derecho Constitucional

Universidad Católica del Norte - Coquimbo

\section{INDICE}

I. Introducción

II. Valor de una conciencia solidaria

III. Grados de integración económica en el continente

IV. Alcances de la institucionalidad universitaria en Chile

V. Áreas específicas del derecho susceptibles de estudios e investigaciones jurídicas conjuntas: Sugerencias

VI. Consideraciones finales

VII. Antecedentes bibliográficos

\section{I.- INTRODUCCION}

Hay muchas interrogantes básicas desde la perspectiva jurídico-académica que sugiere el proceso de integración en nuestra América Latina. Para entender e interpretar este proceso, es necesario enfocarlo en el contexto de las ideas que se han formulado en este sentido en la última década de cara al siglo XXI, análisis que encuentra uno de los terrenos más fértiles en las Universidades Latinoamericanas y por ello siempre será un diálogo sugerente y fructífero.

Es importante la creación de estos espacios de estudio y reflexión que den sentido a los propósitos asociativos, que constribuyan ha otorgar profundidad a este proceso, con capacidad suficiente para internalizarse en el interés colectivo de sus destinatarios: los pueblos latinoamericanos.

Los esfuerzos integracionistas siempre han constituido una oportunidad para la institución universitaria, la que por su naturaleza es centro de reunión, avance, creación y transmisión de conocimientos y como tal, habilitada para proponer soluciones a los 
problemas y carencias que sufren amplios sectores de nuestras poblaciones. Es en este cauce que las Escuelas y Facultades de Derecho deberían reconocer un sitio de acción, traducido en aportes jurídicos sustantivos al desarrollo de latinoamérica.

El Derecho tiene un comportamiento ambivalente ante el desarrollo, que en lo fundamental es concebido como un proceso acelerado y buscado del cambio de las instituciones sociales para hacerlas coherentes con las necesidades, urgencias y derechos de las personas. Por otra parte, es sabido que un sistema social se concreta en instituciones jurídicas, por lo tanto la construcción jurídica, como técnica, está llamada a contribuir a consolidar los nuevos sistemas, advertir y solucionar sus vacíos, eliminando sus contradicciones. La construcción jurídica, es en esencia una disciplina de estabilidad y la concurrencia de esta disciplina es de hecho inevitable en la consolidación del proceso integrador de nuestras naciones.

El Derecho tiene además asignada otra misión, buscar conscientemente el cambio, en la medida que el juicio que le merezca la realidad lo impulse a nuevas concepciones, a la postulación o relevancia de nuevos valores vinculados con la exigencia de nuestro tiempo con la mira de proteger y elevar la dignidad humana. Es por esto por lo que, las Escuelas de Derecho en nuestro mundo latinoamericano, deben unirse en esta tarea común, pudiendo constituirse en una fuerza de proyecciones internacionales por la vía de asumir proyectos académicos conjuntos, en áreas de interés común que por su calidad académica sustenten políticas de desarrollo impulsadas o materializadas por entes del sector público o privado, consiguiendo a su vez recursos financieros adecuados para su consecución.

Este análisis no ignora los esfuerzos y progresos llevados a cabo por las universidades latinoamericanas, en pro del ideal integracionista, el propósito es constribuir a su avance, teniendo en cuenta las nuevas realidades sociales y económicas. En Chile por ejemplo, a partir de 1981 se impuso una profunda reforma de índole institucional en la Educación Superior que dió lugar al surgimiento de universidades estatales y privadas de caracteres nacionales, pero territorialmente emplazadas en diferentes regiones del país, cuya autonomía les ha permitido un desarrollo apreciable, con una clara raigambre regional y con capacidad para relacionarse internacionalmente.

En el pasado más o menos inmediato han tenido lugar iniciativas, proyectos, investigaciones, intercambios y organizaciones que han sido creadas con fines integracionistas, e ideas y proyectos llevados a la práctica, especialmente por importantes Universidades de larga tradición histórica en nuestro Continente.

El Profesor Francisco Orrego Vicuña, decía hace tres decenios: "El proceso integrativo tiene una enorme incidencia en todos los campos, cualquiera que sea la forma que adopte. Precisamente por esta trascendencia vital, por el sinnúmero de complejos problemas que en torno a él surgen, por ser en esencia un fenómeno de reestructuración internacional y por crear conciencia, mentalidad y visión nueva, el fenómeno integrativo debe ser estudiado y analizado, proyectado y divulgado en todos los ámbitos de la cultura y de la actividad de una sociedad. Esta es la enorme tarea de la universidad 
latinoamericana en torno a la integración. La universidad tiene la misión de proporcionar a su ámbito la solución de los problemas que le aquejan en la función de la ciencia y de la técnica que se desarrolla en su seno, en la que participan investigadores, docentes y alumnos. La Universidad latinoamericana como órgano transmisor y divulgador de la cultura, debe transmitir esta "nueva cultura" que se traduce en una mentalidad distinta de mirar el horizonte de nuestro continente, en un nuevo sentir y en una diferente dimensión intelectual para el análisis y solución de los problemas. Se abandona el concepto provinciano por el cosmopolita; el nacional por el continente; el enclaustrado por el integrado" ${ }^{1}$. Son ideas y propósitos vigentes, no obstante, distinta es la condición de nuestro tiempo; el proceso de globalización tiene un carácter señaladamente cultural, entendiendo el "concepto de cultura como el referido a alguien que crece, que se desarrolla, que observa las circunstancias de su medio ambiente, y las interpreta desde el punto de vista de su autodespliegue como sujeto en el mundo" ${ }^{2}$. Asistimos a fenómenos sociales cargados de expectativas como consecuencia del acelerado desarrollo tecnológico, en el cual el ejemplo de las comunicaciones y su evidente impacto en las relaciones interpersonales nos ilustra sobre las mutaciones que puede experimentar la realidad. El resultado ha sido una mayor información en este ámbito del mundo, con todas las expectativas insatisfechas que desencadenan el conocimiento de un mundo que para grandes masas empobrecidas aparece inalcanzable.

Sin embargo, "el futuro que alguna vez inspiró confianza, es ahora una fuente de confusión. No existe consenso sobre el rumbo que debemos seguir en lo sucesivo. En medio de este clima de desorientación resalta una inquietud: la búsqueda de conocimientos y el esfuerzo por controlar nuestro entorno social y cultural no inspira la misma seguridad de antaño. Pero el problema no radica en la aceleración del cambio propiamente tal sino en nuestra incapacidad para hacer frente a las informaciones sin sufrir una crisis. La tradición intelectual que hemos heredado concibe el futuro como algo que puede predecirse, para lo cual sólo basta adquirir los conocimientos suficientes. Aunque ella ha sido una fuente esencial de estabilidad en el pasado, hoy aparece como una fuente de rigidez". Cuando el mundo cambia en forma repentina, las predicciones pueden quedar obsoletas de la noche a la mañana. Los planes basados en estos vaticinios no logran adaptarse a circunstancias variables, por lo que necesitamos encontrar otra fuente de estabilidad que nos permita afrontar los cambios de manera más flexible. ${ }^{3}$

La reorientación de la economía hacia el libre mercado están reflejando una realidad desconocida en el pasado.

La meta se dirige hacia el "Desarrollo sostenible", lo cual significa que no debería consumirse nada o ninguna cosa que no pueda ser reemplazada o renovada en un plazo razonable, así lo formula la Agenda 21 del Programa de Acción que adoptó la

\footnotetext{
${ }^{1}$ ORREGO VICUÑA Francisco. La Integración Política. Edit. Jurídica de Chile, pp. 356 y 357.

${ }^{2}$ MORANDÉ Pedro. America Latina: Los Desafíos de la modernidad. Cuaderno 5 Alborada, pp. 3.

${ }^{3}$ FLORES Fernando. Nuevos principios para un mundo empresarial en cambio, Estudios Públicos. Invierno 1992, pp. 7 .
} 
Conferencia sobre Medio Ambiente y Desarrollo de las Naciones Unidas en Río de Janeiro en 1992.

Este es el contexto en el cual hemos querido situar los desafíos conjuntos que deberían asumir las Escuelas de Derecho en el ámbito internacional latinoamericano.

\section{II.- VALOR DE UNA CONCIENCIA SOLIDARIA}

Es un hecho el rol preponderante de la empresa en la economía de mercado como asignador de recursos, la importancia de la libre competencia, como asimismo, el fortalecimiento que ha adquirido la propiedad privada y la libre creatividad en la economía. Estados que se distancian de su intervención directa en la economía, lo que no significa su prescindencia, especialmente en materias sociales, circunscribiendo su influencia a la fijación de principios y reglas a la que deberán atenerse los actores privados en la producción e intercambio de recursos.

Lo anterior, sin embargo, ocurre en una sociedad caracterizada por la falta de justicia y equidad en amplios sectores de la población, en un ambiente, que en vez de confianza y seguridad, genera inquietud.

Gilles Lipovetsky, a este respecto ha dicho "el mundo en el cual nosotros estamos tiene muchas cosas positivas, pero hay enormes problemas y, a mi modo de ver, este problema del lugar de los individuos que no son buenos para la competencia es el problema fundamental del futuro. Todos no podrán ser grandes jefes de empresa, todos no serán financistas o investigadores. Entonces qué van a hacer los demás, cuál será el lugar que tendrá en nuestra sociedad, que haremos con la gente sencilla. Los padres de familia lo saben, están tan obsesionados por sus hijos, pagan sus estudios, porque saben muy bien que los que no puedan entrar en este mundo de la competencia individualista quedarán rechazados, excluidos, y el mundo entonces será muy duro para ellos.

De ahí el problema que se plantea a partir de ahora: el lugar que ocupará la solidaridad en el mundo de la globalización y la competencia". ${ }^{4}$

En este orden de ideas tiene particular valor la reflexión del Santo Padre Juan Pablo II, en "Sollicitudo rei socialis", cuando señala: "la solidaridad no es, un sentimiento superficial por los males de tantas personas excluidas, sean cercanas o lejanas, al contrario, es una determinación firme y perseverante de empeñarse por el bien común, es decir, por el bien de todos y de cada uno para que todos seamos responsables de todos. Esta determinación se funda en la firme convicción de que lo que frena el pleno desarrollo es el afán de ganancias y la sed de poder. Tales actitudes y estructuras solamente se vencen con la ayuda de la gracia divina, mediante una actitud diametralmente opuesta, esto es, la entrega por el bien del prójimo, que se está dispuesto a "per-

4 LIPOVETSKY GILLES, "Postmodernidad y revolución individualista" : Profesor visitante de la Universidad de la New York; Universidad Andrés Bello, El Mercurio, Agosto, 16 de 1998. 
derse" por el otro, en lugar de explotarlo, y a servirlo, en lugar de oprimirlo para el propio provecho".

Esta actitud a la que se refiere el Sumo Pontífice, debe dar lugar a una "cultura solidaria", que si bien debe comenzar en la familia, su cultivo esmerado también ha constituirse en una responsabilidad para las universidades de nuestro continente.

La economía de mercado no puede alejarse o distanciarse de los esfuerzos por lograr que ella sea socialmente justa, se trata sin duda de decisiones políticas, esto es, de quienes ejercen el poder político, sin que ello exima a las universidades de su concurso. En este caso las Escuelas de Derecho, tienen un compromiso importante, por cuanto en su seno se prepara una buena parte de la clase dirigente cuya influencia ha sido siempre gravitante en el destino de nuestras naciones. Estas instituciones formadores de los hombres de derecho deben proporcionar respuestas doctrinarias en las que ha de fundarse la legislación integracionista, especialmente en relación con el futuro del trabajo, actividad irremplazable para elevar la dignidad de la persona humana.

Es preciso intensificar la creación de zonas o áreas de convergencia haciendo del diálogo una práctica habitual, fundada entre otros muchos vínculos en el intercambio de estudios y de experiencias. Es necesario invertir con esperanza en lo que es propio de la naturaleza del quehacer universitario: "en el alma de las personas". Esta cultura solidaria asegurará en el futuro que en las decisiones políticas, económicas y sociales que adopten las nuevas generaciones, primen los criterios éticos, sociales, culturales y espirituales, como resultado de esta nueva forma de convivencia. Es de este modo que las Escuelas de Derecho en la América Latina cumplirán el rol que le exige nuestro tiempo.

A estos propósitos el preámbulo del proyecto de declaración mundial sobre la Educación Superior en el Siglo XXI: Visión y Acción, de la Conferencia Mundial sobre estas instituciones, de la Organización de las Naciones Unidas para la Educación, la Ciencia y la Cultura, dice en el primero de sus párrafos: "en los albores del nuevo siglo, se observan una demanda de educación superior sin precedentes, acompañada de una gran diversificación de la misma, y una mayor toma de conciencia de la importancia fundamental que este tipo de educación reviste para el desarrollo sociocultural y económico y para la construcción del futuro, de cara al cual las nuevas generaciones deberán estar preparadas con nuevas competencias y nuevos conocimientos e ideales. La educación superior comprende "todo tipo de estudios, de formación o de formación para la investigación en el nivel postsecundario, impartidos por una universidad $\mathrm{u}$ otros establecimientos de enseñanza que estén acreditados por las autoridades competentes del Estado como centros de enseñanza superior" 5 .

Estos nuevos conocimientos e ideales deben cimentarse en la solidaridad, que significa "sentir juntos".

5 Definición aprobada por la Conferencia General de la UNESCO en su $27^{\circ}$ reunión (noviembre de 1993) en la Recomendación sobre la convalidación de los estudios, títulos y diplomas de enseñanza superior. 
Es el ejercicio de la libertad con otra óptica. "una libertad a la altura de la dignidad humana es una libertad solidaria. Mi libertad no termina donde comienza la tuya; tal idea proviene de la concepción del hombre como ser espacial. Mi libertad se abre a la tuya, se enlaza con ella de tal modo que mutuamente se potencian en un dinamismo cuyo parámetro decisivo no es el espacio sino el tiempo". Es la libertad social la única instancia capaz de generar un poder justo", 6

\section{III.- GRADOS DE INTEGRACIÓN ECONÓMICA EN EL CONTINENTE}

Las consideraciones precedentes nos conducen a un escenario internacional que ha experimentado importantes modificaciones. La acentuada globalización señalada conlleva un aumento sustantivo en el volumen del comercio internacional, cobrando un dinamismo importante la inversión extranjera directa y una mayor participación de los servicios, tanto en su producción como en sus prestaciones transfronterizas.

En el Continente Americano han proliferado acuerdos comerciales que admiten distintos grados de integración económica: el Tratado de Libre Comercio de América del Norte (NAFTA); el Mercado Común del Sur (MERCOSUR); el grupo de los tres (México, Colombia y Venezuela); la reactivación del acuerdo de Cartagena y de los existentes en Centroamérica y en el Caribe, además de múltiples pactos de carácter bilateral en el marco de ALADI. Asimismo, como resultado de la Cumbre de Miami, de diciembre de 1994, se iniciaron negociaciones que dieron origen al Acuerdo de Libre Comercio de las Américas (ALCA).

Chile ha desarrollado una estrategia comercial de negociación regional y bilateral. Con este propósito se han suscrito diversos acuerdos de libre comercio y de complementación económica bilateral con Argentina, México, Bolivia, Colombia, Ecuador y Venezuela. Nuestro país persigue un modelo de regionalismo abierto como principio básico de su estrategia de negociaciones económicas internacionales. Su política exterior se orienta de manera prioritaria hacia Latinoamérica. Se trata, en efecto, del ámbito natural de referencia y de acción externa, donde coinciden tanto sus intereses políticos y culturales como importantes vínculos económicos.

En los años recientes se constatan más avances en el campo de la integración latinoamericana que durante los últimos cincuenta años. El comercio intrarregional ha aumentado sustantivamente, y esto se ha logrado muchas veces desbordando los marcos establecidos en los acuerdos vigentes. Las inversiones se incrementan sustantivamente sin esperar la vigencia de los respectivos tratados para su comercio de servicio, incluso antes de establecerse normativas específicas que lo regulen. ${ }^{7}$

6 LLANOS CIFUENTES Alejandro. Etica y Democracia. Alborada cuaderno 3, pp. 10.

7 Acuerdo de Complementación Económica Chile-Mercosur. Ministerio de Relaciones Exteriores. Dirección General de Relaciones Económicas Internacionales. 
La realidad suscintamente transcrita, permite afirmar otra, los inexplicables vacíos en relación con la modernización de las instituciones jurídicas para una aplicación eficiente como instrumentos al servicio del proceso de desarrollo latinoamericano.

La sustentación jurídica, de las diversas iniciativas, acuerdos y proyectos se encuentran con serias dificultades de carácter legal al momento de dar curso operativo a su implementación específica.

Es en esta instancia, en que es posible sostener que las Escuelas de Derecho tienen un campo de acción conjunta, en el cual deberían perseverar, concitando acuerdos biląterales o multilaterales entre ellas, con la mira de realizar aportes jurídicos en dicha dirección, contribuyendo de manera práctica a hacer oír su voz en una materia de suyo importante, convirtiéndose de este modo en un instrumento eficaz al servicio del desarrollo en nuestro continente. El resultado puede ser bastante concreto; la formación de profesionales del derecho aptos en el manejo de la legislación latinoamericana, ello ayudará a superar numerosos problemas de índole legal. Es evidentemente una tarea ambiciosa, que duda cabe, y no puede alcanzarse en un plazo breve; pero sí pueden darse pasos importantes orientados en este sentido. Se trata en un comienzo de aunar voluntades y al mismo tiempo construir buenas ideas, que sí son tales, los recursos necesarios para hacerlos realidad no son un imposible.

\section{IV.- ALCANCES DE LA INSTITUCIONALIDAD UNIVERSITARIA EN CHILE}

El fomento del desarrollo de la educación en todos los niveles, el estímulo de la investigación científica y tecnológica, la creación artística y la protección e incremento del patrimonio cultural de la Nación es asumido por el Estado de Chile como un deber, y así lo consigna de manera expresa a través de una ley orgánica complementaria de la Constitución.

Es dentro de este ámbito y en el contexto por una parte del reconocimiento y amparo a los grupos intermedios a través de los cuales se organiza y estructura la sociedad garantizando su autonomía y por otra de la libertad de enseñanza, en que es posible sustentar la internacionalización de la universidad chilena.

La institución universitaria, encuentra también su amparo constitucional en nuestro país, en la citada libertad de enseñanza, garantía constitucional que integra junto a las libertades de conciencia y de cultos y de información, lo que se denomina "Protección del pensamiento libre en cuanto al bien jurídico protegido" ${ }^{8}$.- Nuestra Carta Política, consagra dicha libertad en el artículo $19 \mathrm{~N}^{\circ} 11$, en términos tales, que ésta incluye el derecho de abrir, organizar y mantener establecimientos educacionales, sin otras limitaciones que las impuestas por la moral, las buenas costumbres, el orden público y la seguridad nacional. Expresamente previene que la enseñanza reconocida

8 EVANS DE LA CUADRA Enrique. Los Derechos Constitucionales. Tomo I pp 255 
oficialmente no podrá orientarse a propagar tendencia político-partidista alguna. Los padres tienen el derecho de escoger el establecimiento de enseñanza para sus hijos.

Una ley orgánica constitucional establecerá los requisitos que deberán exigirse en cada uno de los niveles de la enseñanza básica y media y señalará las normas objetivas de general aplicación, que permita al Estado velar por su cumplimiento.

Para referirse a la Educación Superior, el constituyente establece que dicha ley orgánica del mismo modo, establecerá los requisitos para el reconocimiento oficial de los establecimientos de "todo nivel" 9 -

Fundado en este precepto, el Estado reconoce oficialmente a las Universidades, además de otras instituciones de educación superior y distingue ya ha sido señalado entre universidades estatales y privadas, las primeras solo pueden crearse por ley, en tanto que las últimas, serán siempre Corporaciones de derecho privado sin fines de lucro.

La autonomía universitaria la define el legislador, como "el derecho de estos establecimientos a regirse por sí mismo de conformidad con lo establecido en sus estatutos en todo lo concerniente al cumplimiento de sus finalidades y comprende la autonomía académica, económica y administrativa" ${ }^{10}$.

Con anterioridad a la Ley 18.962 Orgánica Constitucional Enseñanza, el D.F.L. $N^{\circ} 1$ de Educación de 1980 definió la Universidad desde la perspectiva legal como "una institución de educación superior, de investigación, raciocinio y cultura que, en el cumplimiento de sus funciones, debe atender adecuadamente los intereses y necesidades del país, el más alto nivel de excelencia". El mismo D.F.L. $N^{\circ} 1$ agrega que "corresponde especialmente a las universidades:

a) Promover la investigación, creación, preservación y transmisión del saber universal y el cultivo de las artes y de las letras.

b) Contribuir al desarrollo espiritual y cultural del país de acuerdo con los valores de su tradición histórica.

c) Formar graduados y profesionales idóneos, con la capacidad y conocimientos necesarios para el ejercicio de sus respectivas actividades.

d) Otorgar grados académicos y títulos profesionales reconocidos por el Estado.

e) En general, realizar las funciones de docencia, investigación y extensión que son propios de la tarea universitaria" ${ }^{11}$.

\footnotetext{
${ }^{9}$ Constitución Política de la República de Chile de 1980, art. 19 №11.

${ }^{10}$ Ley N 18.962 Orgánica Constitucional de Enseñanza. arts. 29,30,74.

${ }^{11}$ D.F.L N 1 de Educación de 1980 , arts. 1 y 2.
} 
Ha de agregarse que en Chile la libertad de enseñanza, se encuentra vinculada con el derecho a la educación, garantía constitucional, que preceptúa que "la educación tiene por objeto el pleno desarrollo de la persona en las distintas etapas de su vida".

Establece que la educación básica es obligatoria, debiendo el Estado financiar un sistema gratuito con tal objeto, destinado a asegurar el acceso a ella de toda la población.

Corresponderá al Estado, asimismo fomentar el desarrollo de la educación en todos sus niveles; estimular la investigación científica y la protección e incremento del patrimonio cultural de la Nación. cación ${ }^{12}$ -

Es deber de la comunidad contribuir el desarrollo y perfeccionamiento de la edu-

El campo de su autonomía, especialmente la académica, concebida como "la potestad para decidir por sí mismas la forma cómo se cumplan sus funciones de docencia, investigación y extensión y la fijación de sus planes y programas de estudios", permite que en el ejercicio de sus funciones, su acción trascienda el ámbito nacional, para situarse de manera creciente en el variado espectro de la comunidad universitaria internacional.

\section{V.- ÁREAS ESPECÍFICAS DEL DERECHO SUSCEPTIBLES DE ESTUDIOS E IN- VESTIGACIONES JURÍDICAS CONJUNTAS: SUGERENCIAS}

El propósito de estas sugerencias, es sólo dejar en evidencia, que aunando voluntades en torno a un interés común, es posible que las Escuelas de Derecho del Continente de manera práctica puedan relacionarse con claras ventajas, incluidas experiencias en los métodos de enseñanza e investigación coadyuvando entre sí, de este modo, a su modernización.

Es posible hacerlo en todas las disciplinas que configuran su quehacer vinculándolo a la integración de los pueblos en latinoamérica.

Solo a modo ejemplar, consignamos la síntesis siguiente:

- Constitucionalización del derecho civil en relación con el Recurso de Protección, institución existente en nuestro ordenamiento jurídico.

- Arbitraje comercial internacional.

- Estudios referidos a la contratación comercial moderna.

- Problemas que se presentan en el transporte terrestre, marítimo y aéreo en los países latinoamericanos.

${ }^{12}$ Constitución Política cit., art $10 \mathrm{~N}^{\circ} 10$. 
- Estudios comparados sobre el Estatuto del Inversionista extranjero.

- Estudios comparados en materias de derecho ambiental.

- Incorporación de las actividades del seguro y del reaseguro a las reglas de la economía de mercado.

- Estudio comparado de leyes antimonopolio.

- Materias sobre operaciones de cambio y comercio exterior.

- En temas constitucionales, pueden incluirse estudios comparados en materias de derechos económicos-sociales. Los llamados Derechos de la Tercera Generación (protección supranacional de los recursos naturales, organizaciones de la comunidad internacional para detener el genocidio, el derecho a la seguridad colectiva). El Orden Público Económico; Justicia Electoral; Tribunales Constitucionales; Institucionalidad de los partidos políticos; debate teórico sobre la Democracia en Latinoamérica.

- Sistemas procesales penales.

- Estudios sobre el regionalismo americano, en cuanto a la formación y protección de los derechos.

- Estudios, investigación y análisis de instrumentos internacionales regionales en materias de derechos humanos.

- Estudios, investigaciones y análisis sobre derecho del mar.

- Estudios, investigaciones y análisis referidos a la erosión, desechos tóxicos, destrucción de la bióteca en relación con la población de América Latina.

- En materia minera, estudios de derecho comparado referidos a la propiedad minera, régimen de concesiones, amparo, contratos.

- En el derecho inmobiliario, estudios comparados de legislación sobre urbanismo y construcciones. Sistemas sociales de construcción de viviendas. Normas crediticias para adquisición de viviendas, incluido el sistema bancario.

- En materia penal, estudio de legislación comparada sobre tráfico de estupefacientes y su vinculación con el lavado de dinero. Estudios con un enfoque global y multidisciplinario del consumo de droga como fenómeno cultural.

- Estudio comparado sobre la Propiedad Intelectual e Industrial, en sus aspectos jurídico-económicos.

- La transferencia de tecnología y los problemas jurídicos involucrados.

- Problemas jurídicos no resueltos en relación con la Informática.

- Las referencias temáticas enumeradas y sin duda muchas otras, pueden ser objeto de análisis, investigaciones y estudios, en conferencias inter-escuelas, seminarios 
conjuntos aprovechando las formas modernas de comunicación; jornadas interdisciplinarias; académicos invitados; congresos, revistas y otras publicaciones, video conferencias y en general recurriendo a las variadas alternativas que ofrece la actual difusión del conocimiento.

\section{VI.- CONSIDERACIONES FINALES}

Un interesante análisis del novelista, ensayista e historiador Héctor Aguilar Camín, reproducido por la "Revista Estudios Públicos" de Chile, de 1994, extraído de su libro "Subversiones silenciosas: Ensayos de historia y política de México", consigna al final de sus reflexiones, llenas de contenido y amor a su Patria: "los mexicanos de hoy son más mexicanos que nunca". Por razones de integración de las comunicaciones y generalización de la escuela en la era de la mayor influencia norteamericana de México, es decir, en las últimas cinco décadas, México ha alcanzado la mayor uniformidad cultural y la mayor cohesión nacional que haya tenido nunca. Aún así, se pregunta: Cuál será la suerte del nacionalismo y de la identidad nacional de México. Es imposible predecir nada, salvo que, hoy como ayer, las señas de identidad mexicanas no permanecerán inmutables, nadie podrá petrificarlas en sus hallazgos pasados. Los cambios acumulados en el país y los que impone la globalización del mundo desafían nuestras antiguas certezas. Pero la gestión nacional mexicana ha sido larga y nada de lo sedimentado en ella se evapora fácilmente, al contacto con los otros, porque nada tampoco llegó ahí de pronto y como al azar, sino a través de largos procesos de destilación simbólica, que ninguna influencia epidérmica puede suplantar.

No obstante, la crisis de certidumbre sobre el futuro deseable de esa conciencia nacional deberá inducirnos a reconocer la rica pluralidad de sus fuentes y a abrir, más que cerrar, el catálogo de sus inclusiones. Es quizás la hora propicia para pensar generosa, más que defensivamente, nuestras herencias e influencias; para celebrar el contacto y la mezcla con otros, porque ésa es la materia misma de nuestro presente y la inminente obligación de nuestro futuro". ${ }^{13}$

Compartimos con Aguilar Camín, el horizonte que se abre ante nuestra vista en medio de la incertidumbre. Es precisamente a partir de nuestras identidades nacionales, que debemos vivir experiencias comunes, construyendo juntos una auténtica sociedad democrática en latinoamérica, cobijando sus sueños y esperanzas en los fraternos brazos de la solidaridad, fundada en la concepción de que "mi libertad se abre a la tuya, se enlaza con ella, de tal modo que mutuamente se potencian" ${ }^{14}$

13 AGUILAR CAMÍN Héctor en: Estudios Públicos. "La Invención de México. Notas sobre nacionalismo e identidad nacional", (Invierno 1994), pp. 29.

LLANOS C Alejandro. Doc. cit. pp. 10. 


\section{VII.- ANTECEDENTES BIBLIOGRÁFICOS}

1. Constitución Política de la República de Chile. Edición oficial 1998. Editorial Jurídica de Chile.

2. Ley Orgánica Constitucional de Enseñanza N¹8.962.

3. Enrique Evans de la Cuadra. Los Derechos Constitucionales. Tomo I. Editorial Jurídica de Chile, 1999.

4. Ley Orgánica Constitucional de Bases Generales de la Administración del Estado, $N^{\circ} 18.575$.

5. Decreto con Fuerza de Ley $\mathrm{N}^{\circ} 1$ de 1980 del Ministerio de Educación.

6. Mensaje de S.E. el Presidente de la República con el que envía un proyecto de ley marco de universidades estatales.

7. Proyecto de declaración mundial sobre la educación superior en el siglo XXI: Visión y acción. París, 5-9 de octubre de 1998. Conferencia Mundial sobre la Educación Superior.UNESCO.

8. "Sollicitudo rei socialis". S.S. Juan Pablo II. Carta encíclica sobre "La preocupación social de la Iglesia".

9. Acuerdo de Complementación Económica CHILE-MERCOSUR. Ministerio de Relaciones Exteriores. Dirección General de Relaciones Económicas Internacionales.

10. Alejandro Llanos Cifuentes. Ética y Democracia. Alborada. Residencia Universitaria y Centro Cultural. Cuaderno 3.

11. Pedro Morandé. Alborada. América Latina: Los Desafíos de la Modernización.. Residencia Universitaria y Centro Cultural. Cuaderno 5.

12. Conferencia sobre la Enseñanza del Derecho y el Desarrollo. Consejo de Decanos de las Facultades de Derecho (Chile). Instituto de Docencia e Investigaciones Jurídicas. (Chile). Valparaíso. Editorial Andrés Bello, 1971.

13. Gonzalo Rojas S., Marcel Achurra G., Patricio Dussaillant. Derecho Político. Apuntes de clases del profesor Jaime Guzmán Errázuriz. Editorial Universidad Católica de Chile B. 1995

14. José Luis Cea Egaña. El Sistema Constitucional de Chile. Síntesis crítica. Facultad de Ciencias Jurídicas y Sociales. Universidad Austral de Chile, 1999.

15. Hugo Castellón. Venegas. Laura E. Rebolledo Contreras. Aspectos sobre la Constitucionalización del Derecho Civil. Editorial Jurídica Conosur, 1999.

16. Francisco Orrego Vicuña. La Integración Política. Editorial Jurídica de Chile, 1966. 
17. Francisco Orrego Vicuña. Chile y el Derecho del Mar. Editorial Andrés Bello, 1972.

18. Gilles Lipovetsky. "Postmodernidad y Revolución Individualista". El Mercurio, 16 de agosto, 1998.

19. José María Eyzaguirre G. "Cambios experimentados en la profesión legal". de la H. El Mercurio, 21 de noviembre de 1995.

20. "La solidaridad en una economía libre". Revista de Estudios Públicos. Invierno 1999, pág. 291.

21. "Nuevos principios para un mundo empresarial en cambio". Revista Estudios Públicos. Invierno 1992, pág.5.

22. "La Invención de México". Revista Estudios Públicos. Invierno 1994, pág.5.

23. "La identidad latinoamericana". Revista Estudios Públicos. Invierno 1994, pág. 31.

24. "El laberinto de las formas de vida". Revista Estudios Públicos. Primavera 1995. pág. 5.

25. "Bases conceptuales y marco de referencia para la elaboración de políticas ambientales en América Latina". Revista Estudios Públicos Verano 1995, Pág.173.

26. "Desafíos del medio ambiente y respuestas políticas". Revista Estudios Públicos. Primavera 1997.

27. "Una perspectiva ecológica sobre el uso del agua en el Norte Grande". Revista Estudios Públicos. Primavera 1997.

28. "Los países en desarrollo en la Ronda Uruguay". Revista Estudios Públicos. Otoño 1995.

29. "Una lección de la mejor historia". Revista Estudios Públicos. Otoño 1995.

30. "Efectos de la Asociación de Chile al Mercosur en el sector agrícola y agroindustrial". Revista Estudios Públicos. Invierno 1996.

31. "Acuerdos de libre Comercio y exportaciones regionales de Chile". Revista Estudios Públicos. Invierno 1996.

32. "Simetrías y divergencias en la historia de Argentina y Chile". Revista Estudios Públicos. Invierno 1996.

33. "México y el testigo insomne". Revista Estudios Públicos. Primavera 1996.

34. "Educación en artes liberales en el mundo de la tecnología". Revista Estudios Públicos. Primavera 1996.

35. "Notas sobre la educación superior en el Reino Unido, Estados Unidos y el Continente europeo". Revista Estudios Públicos. Primavera 1996. 
36. "Los estudios generales, la sociedad ilustrada y la universidad chilena". Revista Estudios Públicos. Primavera 1996.

37. "La trayectoria latinoamericana o la modernidad". Revista Estudios públicos. Otoño 1997.

38. "Heroísmo y utopía". Revista Estudios Públicos. Verano 1997.

39. "Diversidad Cultural: Una perspectiva universal". Revista Estudios Públicos. Verano 1993.

40. "Chile y la sociedad Post-Industrial". Revista Estudios Públicos. Verano 1986 